\title{
Notas sobre la escritura escolar como efecto de verdad sobre el sujeto o como gesto en la relación con el saber
}

\author{
Notes on School Writing as a Truth Effect on the Subject or as a Gesture in the \\ Subject's Relation to Knowledge
}

\author{
Manuela Cartolari ${ }^{1}$ \\ Universidad de Buenos Aires \\ Buenos Aires, Argentina \\ manucartolari@yahoo.com \\ Jusmeidy Zambrano² \\ Universidad Nacional Experimental del Táchira \\ Venezuela \\ jzambran@unet.edu.ve
}

Recibido 18 de setiembre de 2012 • Corregido 20 de febrero de 2013 • Aceptado 13 de marzo de 2013

Resumen. En este ensayo se analizan presupuestos ético-filosóficos subyacentes a cómo se conciben las escrituras de los alumnos en el ámbito educativo en relación con la subjetividad y el saber. La noción de poder desarrollada por Foucault se vincula a lo escrito en tanto "efecto de verdad" sobre el sujeto y a la concepción reificada de su escritura, entendida como superficie especular del pensamiento. A ello se contrapone el carácter de espaciamiento entre el sí mismo y el signo que implica escribir, espaciamiento en el que Derrida señala que la subjetividad se origina en el sentido producido por la diferencia. Finalmente, se propone pensar la escritura como gesto en la dirección que plantea Agamben, puesto que al sustraerse de la lógica de los medios y los fines, el gesto se pone de manifiesto en la escritura como la comunicabilidad de una comunicabilidad y pura potencia de pensar, es decir, como acontecimiento. Si al escribir un alumno es convocado a producir sentido no solo sobre un conocimiento "puntual", sino sobre su propio ser-en-el-lenguaje como sujeto histórico y deseante, la escritura se asume como gesto hacia el saber $y$, por tanto, emerge en contrapartida la dimensión ético-política acerca de cómo comprendemos, evaluamos y respondemos a sus escritos.

Palabras claves. Subjetividad, escritura, saber, Revista Electrónica Educare, Costa Rica.

1 Doctoranda en Educación, becaria del Consejo Nacional de Investigaciones Científicas y Técnicas (CONICET) de Argentina y docente de la Universidad de Buenos Aires. Participa en el equipo de la Dra. Paula Carlino desde el año 2008 y es integrante del GICEOLEM (Grupo para la Inclusión Educativa a través de Ocuparnos de la Lectura y la Escritura en todas las Materias), se dedica a investigar la lectura y la escritura como herramientas de acceso, participación y apropiación del conocimiento disciplinar en la formación docente en Ciencias Sociales.

2 Profesora de la Universidad Nacional Experimental del Táchira adscrita al Departamento de Ciencias Sociales. Doctoranda en Ciencias de la Educación de la Universidad Nacional de La Plata (Argentina). Especialista en Promoción de la Lectura y Escritura y Licenciada en Educación, mención Castellano y Literatura de la Universidad de Los Andes de Venezuela. Investigadora "A" del Programa de Estímulo a la Innovación e Investigación (PEII). Participa en el equipo de la Dra. Paula Carlino GICEOLEM (Grupo para la Inclusión Educativa a través de Ocuparnos de la Lectura y la Escritura en todas las Materias) desde el año 2011. Las investigaciones que realiza están relacionadas con las prácticas de escritura en las carreras de Ingeniería. 
URL: http://www.una.ac.cr/educare

CORREO: educare@una.cr

\begin{abstract}
This paper discusses ethical and philosophical assumptions on how the writings of students are seen in the education context, in terms of subjectivity and knowledge. Foucault's concept of power is associated to writing as a "truth-effect" on the subject and to the reified concept of the subject's writing, understood this as a mirror surface of thought. This stands in contrast to the spacing between the self and the sign implied in writing; spacing where subjectivity emerges, according to Derrida, in the sense produced by the difference. The conclusion suggests seeing writing as a gesture along the lines proposed by Agamben, because by overcoming the means-ends logic, the gesture becomes evident in the writing as a communication of communicability and of the pure power of thinking, i.e. an event. If, when writing, students are called to produce sense not only on a "specific" area of knowledge, but about their own selves within language as historical and desiring subjects, writing is assumed as a gesture toward knowledge, emerging therefore the ethical-political dimension on how we understand, evaluate and respond to students' writings.
\end{abstract}

Keywords. Subjectivity, writing, knowledge, Educare Electronic Journal, Costa Rica.

\title{
Introducción
}

La escuela ha constituido, desde sus comienzos, el lugar institucional de aculturación en lo escrito de las nuevas generaciones $y$, hasta hoy, que los alumnos aprendan a leer y a escribir vertebra gran parte del quehacer de la escolarización obligatoria. Pero además, si alfabetizar es uno de los fundamentos de la escuela desde su creación, enseñar mediante la escritura otros saberes y conocimientos no lo es menos. En un mundo caracterizado por la tecnologización creciente de la palabra ${ }^{3}$, como sostiene Ong (1997), escribir consiste en un medio por excelencia para conseguir los más variados fines. La escolarización de la escritura plantea, por ello, la necesidad de analizar no solo para qué se utiliza esta herramienta en educación sino, más radicalmente, el sentido que puede adquirir en la relación del sujeto con el saber, como así las diferentes maneras de entender la subjetividad en los procesos de enseñanza y aprendizaje.

\section{La escritura como efecto de verdad sobre el sujeto o como momento de su exteriorización}

Lo que nos sostiene en la inquietud y en el esfuerzo de escribir es la certidumbre de que en la página queda algo que no ha sido dicho.

(Pavese, 1976, p. 185)

3 Walter Ong (1997) sostiene que la escritura - desde la creación de tablas, plumas, códices y hasta las más recientes computadoras- comporta una tecnología en tanto consiste en una actividad mediada por utensilios, técnicas que requieren de práctica y ciertas reglas conscientemente elaboradas que rigen su despliegue. Si bien el objetivo de este trabajo no es profundizar en los efectos de la tecnologización de la oralidad, vale aclarar aquí que el autor concluye que la escritura, al objetivar el habla y librarla de su inmediatez mediante su inscripción en soportes materiales y externos a los sujetos, ha transformado sustancialmente los modos de pensar y expresarse de las sociedades alfabetizadas. 
Si existe algo que a primera vista comparte la categoría de "verdad" con la escritura, es la idea de lo inmanente. La verdad es verdad en cuanto esencia, y la escritura es una marca que permanece. Así como en la verdad, hay en lo escrito algo que trasciende el "aquí y ahora", algo que tal vez nada pueda sintetizar mejor que el proverbio latino "verba volant, scripta manent".

En efecto, escribir, en tanto la letra es aquello del orden del lenguaje que al materializarse se separa del cuerpo (Fontaine, 1995), es en última instancia la marca de una exteriorización. Esa marca puede concebirse como una reificación ${ }^{4}$ del sujeto o, por el contrario, como la huella de un momento en su devenir. Un momento en el que lo humano, como humano dentro del orden del lenguaje, se representa al mismo tiempo que niega la transparencia de su representarse. La escritura, como una marca que al permanecer desvincula al sujeto de su situacionalidad, es opaca, incompleta, impura. Pero a la vez, es una representación exteriorizada mediante la cual el sujeto mismo, y los otros, pueden actuar y operar sobre ese representarse. El sujeto se manifiesta, entonces, como sujeto humano en el lenguaje y en su propia escritura; pero simultáneamente, resulta irreductible a lo que escribe y a la verdad que se quiera pretender que lo escrito expresa sobre él. Agamben (2005), con respecto a la figura del autor, señala acertadamente esta tensión:

Y así como el autor debe permanecer inexpresado en la obra, y sin embargo, precisamente de esta manera, atestigua su propia irreductible presencia, así la subjetividad se muestra y resiste con más fuerza en el punto en que los dispositivos la capturan y la ponen en juego. Una subjetividad se produce donde el viviente, encontrando el lenguaje y poniéndose en juego en él sin reservas, exhibe en un gesto su irreductibilidad a él. (p. 94)

En relación con esta irreductibilidad, nos interesa enfocar aquí ciertos usos de la escritura en educación, que aún hoy se constatan en lo que informan alumnos y docentes. Conforme avanza la escolaridad, escribir suele adquirir, en modo privilegiado, el sentido de "registrar" aquello que debe aprenderse y de "demostrar" al docente lo que se aprendió o no se aprendió (Di Benedetto y Carlino, 2007; Diment y Carlino, 2006; Fernández y Carlino, 2006).

Del mismo modo, la lectura de lo que los alumnos escriben por parte de sus educadores responde frecuentemente al propósito de efectuar operaciones de sanción: el alumno escribe y el docente califica, aprueba o desaprueba. Sin pretender negar la necesidad de prácticas evaluativas en educación, ni mucho menos sostener que no debería evaluarse el aprendizaje

4 Se hace referencia al término «reificación» acuñado por Honneth (2007) para designar el “(...) comportamiento humano que quebranta nuestros principios morales o éticos en tanto otros sujetos no son tratados de acuerdo con sus cualidades humanas, sino como objetos insensibles, inertes, como 'cosas' o'mercancías (...)" (p.17). 
URL: http://www.una.ac.cr/educare

CORREO: educare@una.cr

mediante escritos, sostenemos aquí que existe un sentido de la escritura en la escuela en el que prevalece la producción de "efectos de verdad" sobre los sujetos, por cuanto el saber o no saber del alumnado suele homologarse a lo por él o ella escrito.

Tomamos aquí el concepto de "efectos de verdad" en el sentido planteado por Foucault (1992), es decir, como uno de los elementos de base del funcionamiento reproductivo del sistema político social. Desde esta perspectiva, junto al derecho como norma, los "juegos de verdad" se movilizan para el sostenimiento del poder a partir de la producción de "efectos de verdad" en los discursos sobre el mundo.

En esta línea de análisis, la escuela, como las demás instituciones, es una conformación social que se encuentra obligada a producir verdad desde el poder que la exige. Nuestra tesis, en este punto, consiste en que las formas de concebir la escritura de los alumnos y alumnas ${ }^{5}$ pueden articularse y viabilizar, en parte, esta producción de efectos de verdad, puesto que, como se ha señalado, una de las maneras corrientes de entender a la escritura consiste en tomarla más como una imagen/reflejo del pensamiento del alumnado, que como un momento del sujeto en el que se expresa la tensión de su ser-en-el-lenguaje, en la dirección que plantea Agamben (2005).

En cierto sentido, la ecuación moderna pensar = ser puede rastrearse cuando el alumno es "dicho" por su escritura, esto es, cuando en lo que escribe queda "demostrado" lo que sabe, lo que piensa y, en definitiva, lo que es (inteligente, estudioso, buen o mal alumno, socialmente desfavorecido, falto de "capital cultural", etcétera).

Sabemos que la escuela del llamado modelo tradicional orbitó alrededor de la soberanía del sujeto cartesiano, por lo que la reducción de la escritura de los alumnos a una esencia o a una pretendida verdad sobre la subjetividad se vincula a un paradigma de larga data en el ámbito escolar. Sabemos también que el sujeto moderno -en tanto sujeto trascendentalpiensa y, porque piensa, existe: a través del pensamiento el sujeto garantiza su experiencia objetiva del mundo; un mundo que puede y debe ser decodificado, y sometido por la razón. Los otros, reunidos bajo la también moderna noción de "pueblo", formarán asimismo parte de aquello que puede y debe entrenarse, modificarse o adaptarse.

El Enciclopedismo y el lluminismo abrevaron de esta concepción, que reverberó en la acción educativa de la escuela tradicional, preocupada por garantizar una homogeneización básica del pensamiento de los ciudadanos y, ergo, de sus comportamientos económicos y decisiones políticas. La metáfora del alumno como tabula rasa, o superficie en la que había que borrar conocimientos previos y "contaminantes", para inscribir el saber científico y legítimo, dio buena cuenta de este paradigma (Cullen, 1997).

Desde ya, en la afirmación anterior, los verbos borrar e inscribir no deben parecer casuales; como se verá, estas expresiones relacionadas con lo escrito no remiten aquí a una metáfora banal. De hecho, la escritura fue un buen soporte de la lógica moderna en la escuela.

\footnotetext{
En este trabajo enfocamos el problema de las escrituras que se requieren al alumno y las que éste produce y no, como
} sería también posible, a las escrituras que los agentes educativos realizan sobre, o con respecto a los alumnos. 
Recurriremos al cine para brindar un ejemplo claro de esta afirmación, refiriéndonos a las escenas que inauguran el conocido filme Los cuatrocientos golpes, de Truffaut (1959). En dichas escenas, el joven protagonista Doinel ha sido castigado por su maestro y debe permanecer durante el recreo en un rincón del aula, en reprimenda por haber sido sorprendido con la ilustración de una pin-up girl que un compañero dejara caer en su pupitre durante la clase. Enfurecido, el niño escribe en la pared los siguientes versos: "Aquí el pobre Antoine Doinel / castigado injustamente por el profesorcito / por causa de una pin-up caída del cielo. / Será diente por diente, ojo por ojo". La reacción del profesor, al término del recreo, al descubrirlo, es elocuente e irónica: "bravo", exclama dirigiéndose a los demás alumnos, "tenemos un nuevo poeta en nuestra clase, solo que no sabe distinguir un alejandrino de un endecasílabo". Tras esta reprimenda, le ordena al niño borrar su escritura y conjugar por escrito para el día siguiente en tiempo indicativo, subjuntivo y condicional "yo degradé las paredes de la clase, y maltraté la prosodia francesa".

Como puede verse, esta escena, más allá de su humorística crítica a los tiempos en que los castigos emocionales (y en ocasiones físicos) se encontraban legitimados en la escuela, permite notar el uso de la escritura como medio para disciplinar el pensamiento o comportamiento del alumno, en respuesta a su uso para expresar resistencia. Así pues, la secuencia resulta ilustrativa con respecto a cómo se actualizan en la institución escolar, en el sutil interjuego del poder, las ilegalidades y el disciplinamiento a través de la escritura.

Al hablar de poder, nos interesa puntualizar que lo hacemos desde la conceptualización propuesta por Foucault (1992), puesto que ofrece la ventaja de enfocar la construcción de subjetividad en un nivel microfísico, sin por ello desligarse de su concatenación con las estructuras sociales de dominación. El autor propone leer al poder en clave de "táctica y estrategia", atendiendo las relaciones impersonales que se tejen entre individuos o grupos. En este sentido, los efectos de poder, más que reprimir una subjetividad que les sería anterior y externa, definen y reparten papeles que producen a los sujetos en ese mismo proceso:

Entre cada punto del cuerpo social, entre un hombre y una mujer, en una familia, entre un maestro y su alumno, entre el que sabe y el que no sabe, pasan relaciones de poder que no son la proyección pura y simple del gran poder del soberano sobre los individuos; son más bien el suelo movedizo y concreto sobre el que ese poder se incardina, las condiciones de posibilidad de su funcionamiento. (...) En general, creo que el poder no se construye a partir de "voluntades" (individuales o colectivas), ni tampoco se deriva de intereses. El poder se construye y funciona a partir de poderes, de multitud de cuestiones $y$ de efectos de poder. Es éste el dominio complejo que hay que estudiar. Esto no quiere decir que el poder es independiente, y que se podría descifrar sin tener en cuenta el proceso económico y las relaciones de producción. (p. 167-168)

Decir que las relaciones de poder son el "suelo movedizo y concreto" en el que pueden verificarse ciertos efectos de poder, nos advierte del riesgo de realizar una interpretación 
URL: http://www.una.ac.cr/educare

CORREO: educare@una.cr

demasiado simple de la dominación y del papel de la escritura en el contexto escolar. "No existen relaciones de poder sin resistencias; éstas son más reales y más eficaces cuando se forman allí mismo donde se ejercen las relaciones de poder; la resistencia al poder no tiene que venir de fuera para ser real, pero tampoco está atrapada por ser compatriota del poder" (Foucault, 1992, p. 181). Los mecanismos de coacción no son, entonces, tierra firme; existen atravesados por ilegalidades, resistencias, desviaciones. El sujeto se encuentra y se produce, tal vez, en este territorio movedizo del que la escena de Los cuatrocientos golpes, y en general el resto del filme, resulta un acertado ejemplo.

Por ello la escritura, como momento de exteriorización del sujeto, se encuentra más del lado del testimonio de estos movimientos, que del lado de una verdad, testimonio que se sostiene, como apunta Pavese (1976), a partir de la certeza de lo que no podrá decirse. El hecho de que lo escrito no pueda plantearse como una verdad esencial de ese sujeto que escribe resulta, asimismo, y como señala Derrida (1998), de que la escritura, al ser exterioridad, implica un espaciamiento entre el sí mismo y el signo. En este espaciamiento no hay plenitud posible, sino sentido originado en la diferencia:

Constituyéndolo y dislocándolo simultáneamente, la escritura es distinta del sujeto, en cualquier sentido que se lo entienda. Nunca podría pensarse bajo su categoría; de cualquier manera que se la modifique, que se la afecte de conciencia o de inconsciencia, remitirá, a todo lo largo de su historia, a la sustancialidad de una presencia impasible ante los accidentes o a la identidad de lo propio en la presencia de la relación consigo. (...) Determinar una X como sujeto nunca es una operación de pura convención; en cuanto a la escritura jamás es un gesto indiferente. (pp. 91-92)

Aquello que no puede ser dicho, aquello de lo cual no puede pretenderse un origen puro, como no puede pretenderse un origen pleno de la escritura en la cosa ni en la referencia, es el sujeto. La escritura es, en consecuencia, un gesto en el que, a partir de este espaciamiento, la emancipación del signo llama a la presencia del sujeto retroactivamente. En ese devenir- ausencia y devenir diferencia-en-el-lenguaje, según Derrida (1998), la subjetividad se constituye. La escuela, como lugar público de enseñanza y aprendizaje de saberes y conocimientos, y como práctica social disimétrica que compromete "al poder de enseñar y al deseo de aprender" (Cullen, 1997, p. 58), también es un espacio y un tiempo para la subjetivación.

Como veremos en el siguiente apartado, si aprender es producir sentidos (Cullen, 1997), entonces la escritura en la escuela necesita orientarse en tanto gesto del sujeto en su relación con el saber, en vez de utilizarse para producir una "verdad" sobre el sujeto. 


\title{
La escritura como gesto en la relación con el saber
}

\begin{abstract}
Escribir es intentar saber qué escribiríamos si escribiésemos -sólo lo sabemos después-antes es la cuestión más peligrosa que podamos plantearnos. Pero también es la más habitual. La escritura: la escritura llega como el viento, está desnuda, es la tinta, es lo escrito.
\end{abstract}

(Duras, 1994, p. 56)

La escritura puede considerarse como un gesto subjetivo vinculado al saber en tanto no solamente se pone en juego una "normativa" al constituirse en una lengua, sino que implica un conocimiento del viviente en el lenguaje, un conocimiento que, en cierta medida, le es ajeno. Si "escribir es intentar saber qué escribiríamos si escribiésemos", como sostiene Duras (1994) en el epígrafe que encabeza este apartado, hay un saber que no se alcanza si no es a través del proceso mismo de escribir. La escritura puede pensarse, por tanto, como una operación en la que el espaciamiento entre el sujeto y la huella escrita, al modo de un "point de capiton", como plantea Lacan (1997), constituye una significación que permite situar un instante que resignifica lo pasado y lo futuro en el devenir de un sujeto. El saber que permite el escribir es eso "que sólo sabemos después", y por ello, la escritura de los alumnos y alumnas puede ser entendida como un proceso que siempre es ante todo posibilidad, y posibilidad de aprender a través del gesto de escribir.

Sin embargo, y como sostiene Agamben (2004), la escuela en tanto institución social se conforma a partir de un proceso de desemantización y de suspensión de praxis concretas de su inmediata referencia a lo real. De modo análogo al derecho cuando suspende las prácticas y costumbres cotidianas de los sujetos al fijarlas como norma, podemos pensar que la escuela ha connotado con frecuencia la escritura estudiantil como medio para demostrar un saber que se adquiere antes del escribir, y no mediante el propio acto de escribir. En este sentido, es posible que el exceso de significación, que como sugiere el autor distingue a los procesos de lo instituido, haya llevado a que la escolarización de la escritura en la modernidad se parezca a un "estado de excepción", en el que algunos motivos posibles del escribir (esto es, escribir para saber) se encuentran presentes solo para dar cuenta de su ausencia.

No tan lejos de nuestros días, toda la pedagogía pragmática subsidiaria de los planteamientos de Tyler (1949) que cundió en los años 70, y el desarrollo de planes de estudio definidos por objetivos, se basó en un eficientismo que minimizó la noción de aprendizaje únicamente a las manifestaciones observables del sujeto, y a la escritura como una entre ellas. El "interés técnico"6 que distinguiera Habermas (1994), y que subyace a esta utilización

6 El interés técnico, en la teoría de los intereses cognitivos de Habermas (1994, p. 173), supone la orientación básica hacia la gestión y control del medio, congruente con las ciencias empírico-analíticas, que buscan descubrir regularidades -mediante el método experimental- para explicar, predecir y controlar los procesos objetivos de la naturaleza y la sociedad. En este sentido, el interés técnico presupone el modelo sujeto-objeto y la neutralidad valorativa y, por ende, se ajusta a la teoría cartesiana de sujeto. 
URL: http://www.una.ac.cr/educare

CORREO: educare@una.cr

de la escritura al plantearse un eidos especificado de antemano, tanto para el aprendizaje del alumnado como para lo que se espera que sepa escribir, resulta incompatible con una concepción de la escritura como gesto hacia el saber.

Aquí, entendemos la relación con el saber en la dirección que plantea Charlot (2008, p. 47), cuando afirma que se trata de "la relación con el mundo, con los otros y consigo mismo de un sujeto confrontado con la necesidad de aprender". En esa relación, para este autor, resulta esencial que aparezca el deseo, en el sentido psicoanalítico de la noción. Aunque ese deseo, como se subraya en esta perspectiva, no es en absoluto una construcción individual, sino que surge en una determinada relación que se establece con los otros, con el mundo, con un "contenido de pensamiento" y consigo mismo, en la singularidad de una situación. La relación del sujeto con el saber, por tanto, aún siendo una relación singular se encuentra atravesada por determinantes intersubjetivos, sociológicos, históricos y didácticos.

Ahora bien, ¿qué significa pensar la escritura como gesto en la relación con el saber? Y antes que nada, ¿por qué hablar de la escritura como gesto? Desde la fenomenología, Merleau-Ponty (1973) distingue la acción del gesto: la primera consiste en un comportamiento perceptivo; el segundo, en un comportamiento expresivo que, al inaugurar un sentido, va más allá de su simple presencia como hecho. Mientras que la acción es cerrada y consumada, en el gesto toda una estructura de significación invoca a la interpretación; el gesto se encuentra ligado al sentido de la existencia de un individuo, en un determinado proyecto y contexto.

Agamben (2001) también distingue el gesto del actuar; pero, a diferencia de MerleauPonty (1973), realiza un esfuerzo por precisar de qué modo se produce la transformación de una res en res gesta desde un punto exterior a la lógica de los medios y de los fines. En el análisis que realiza Agamben (2001), el gesto no solo se distingue del actuar, sino también del hacer. La diferencia entre agere y facere, derivada de la ética nicomáquea de Aristóteles, sitúa la praxis allí donde actuar en sí mismo es el fin (agere), y a la poiesis donde existe el hacer hacia un fin (facere). Para Agamben (2001), sin embargo, el gesto pertenece a otro orden de la acción, semejante al que se reconoce, por ejemplo, en el cine o en la danza. Estas dos esferas de la acción humana ilustran cómo el gesto presenta unos medios que, como tales, se sustraen al ámbito de la medialidad, sin por ello convertirse en fines:

Una finalidad sin medios es tan desconcertante como una medialidad que sólo tiene sentido con respecto a un fin. Si la danza es gesto es, precisamente, porque no consiste en otra cosa que soportar y exhibir el carácter de medios de los movimientos corporales. El gesto es una medialidad, el hacer visible un medio como tal. Hace aparecer el-ser-en-unmedio del hombre, y de esta forma, le abre su dimensión ética. (p. 54)

Del mismo modo que la danza es gesto cuando, más allá de comunicar la historia que representa o una dimensión estética, comunica por sobre todo el carácter de medialidad de sus movimientos, entender la escritura como gesto la sitúa en el más allá de dejar entrever un conocimiento, una 
"verdad" o un saber preexistente del alumno. Al implicar la comunicación de una comunicabilidad, que al hacerse presente muestra el ser-en-el-lenguaje de la persona, en el gesto de escribir, como reflexiona Agamben (2001), no se produce ni se actúa, sino -por el contrario- se asume y se soporta. Tal vez sea debido a ello que Duras (1994) expresa que "escribir es la cuestión más peligrosa que podamos plantearnos" (p. 56), al mismo tiempo que "la más habitual" (p. 56).

En la escuela, asumir la escritura de los alumnos y alumnas como gesto significa, por tanto, abrir la posibilidad de que un simple hecho, un hacer para un fin, se convierta en acontecimiento. Y como acontecimiento, la escritura posibilita la apertura de la dimensión ética y política del sujeto en educación, en tanto a través de lo que escribe se le convoca a producir sentido no solo sobre un conocimiento o "contenido" puntual, sino sobre su propia relación con el saber como sujeto deseante, y como sujeto histórico en su ser-en-el-lenguaje.

\title{
¿Escribir en la escuela es un acto natural y transparente, o una apuesta ética a la posibilidad y la potencia del sujeto que es- siendo-en-el-lenguaje?
}

\begin{abstract}
Pensar no significa sólo ser afectados por esta o aquella cosa, por este o aquel contenido de pensamiento en acto, sino ser a la vez afectados por la propia receptividad, hacer la experiencia, en cada pensamiento, de una pura potencia de pensar. (Agamben, 2001, p. 18)
\end{abstract}

Si la escritura supone siempre la exteriorización, en términos de espaciamiento, de un momento de la existencia del sujeto en el lenguaje, entonces, en un escrito no es posible dejar testimonio acabado del ser, puesto que es justamente en ese gesto que modula la distancia entre el signo y el sí mismo que el sujeto se produce. Por ello, la escritura deja siempre el testimonio de un saber que no se sabe, ni sobre el sujeto que escribe, ni sobre aquello sobre lo que se escribe. La escritura es, de este modo, un momento para significar y resignificar; en el espacio que se abre entre el sí mismo y el signo, escribir habilita el lugar necesario para el trabajo y la invención entre lo que hay y lo que no hay en esas marcas que se trazan.

Plantear esto en el ámbito de la educación implica revisar qué usos de la escritura se promueven que realicen los alumnos en la escuela. ¿Escriben para alcanzar un saber sobre un momento de su proceso de aprendizaje? ¿Para entrar en diálogo con su propio pensamiento y el de sus pares y docentes mediante sus escritos? ¿Para aprender a través de lo que escriben? ¿Lo hacen para ser evaluados? ¿Se retoma lo que han escrito para que reescriban, relean, o únicamente se les requiere que "expresen por escrito" un saber que se supone ya adquirido y acabado cuando lo hacen?

Estas preguntas, como hemos intentado mostrar aquí, no pueden fundamentarse desde el interés por los procesos cognitivos que intervienen en el aprendizaje, o por mejorar la calidad educativa en términos de alcanzar mayor eficiencia y eficacia en la transmisión de un legado cultural o, aun más, de ampliar la retención en los llamados "sectores con riesgo educativo". Más 
URL: http://www.una.ac.cr/educare

CORREO: educare@una.cr

allá de esas preocupaciones, siempre necesarias y pertinentes de discutir, las maneras de concebir la escritura también conciernen a un debate sobre su dimensión ética y política en educación.

Ética, en tanto escribir -como las demás acciones y procesos singulares de la vida- no es nunca un "acto natural ni prefijado, sino siempre posibilidad y potencia" (Agamben, 2001, p. 18). Y política, en tanto la escuela -como un espacio público para la socialización y la subjetivación mediante la transmisión de saberes y conocimientos (Cullen, 1997)- también es el lugar privilegiado para el acceso a la escritura y a la producción de sentidos mediante el escribir. Por tanto, cabe preguntarnos qué posicionamiento ético-político se asume cuando se "comprende", se "evalúa" o responde (o no) a los escritos de los alumnos y alumnas en educación.

En dirección a ese interrogante, hemos planteado que la escritura del alumnado puede reducirse a viabilizar la producción de "efectos de verdad" sobre los sujetos cuando se la entiende como la superficie de un espejo que refleja el pensamiento, conocimiento o saber ya adquirido. En contraposición, concebir la escritura como gesto es entenderla antes que nada como la comunicabilidad de una comunicabilidad, es decir, como posibilidad y potencia de pensar, conocer y saber. Pensar para escribir y escribir para saber después es, por tanto, un gesto subjetivo que convoca la experiencia del ser como posibilidad y como inacabamiento, aun en el caso de este mismo escrito.

\section{Referencias}

Agamben, G. (2001). Medios sin fin. Notas sobre la política. Valencia, España: Pre-textos.

Agamben, G. (2004). Estado de excepción. Buenos Aires: Adriana Hidalgo.

Agamben, G. (2005). Profanaciones. Buenos Aires: Adriana Hidalgo.

Charlot, B. (2008). La relación con el saber, formación de maestros y profesores, educación y globalización. Cuestiones parala educación de hoy. Montevideo: Trilce.

Cullen, C. A. (1997). Críticas de las razones de educar. Temas e filosofía de la educación. Buenos Aires: Paidós.

Derrida, J. (1998). De la gramatología (Trad O. Del Barco y C. Ceretti). México: Siglo XXI.

Di Benedetto, S. B. y Carlino, P. (2007). Correcciones a exámenes escritos en la universidad: cómo son y para qué sirven a los alumnos. En Memorias de las XIV jornadas de investigación en psicología y tercer encuentro de investigadores en psicología del Mercosur (Tomo I, pp. 273-275). Buenos Aires: Facultad de Psicología de la Universidad de Buenos Aires. Recuperado de http://www.fvet.uba.ar/postgrado/especialidad/blb/Di_Benedetto_ Carlino_Correcciones_examenes_escritos_07.pdf

Diment, E. y Carlino, P. (2006). Perspectivas de alumnos y docentes sobre la escritura en los primeros años de universidad. Un estudio exploratorio. En Memorias de las XIII jornadas de investigación en psicología y segundo encuentro de investigadores en psicología del Mercosur. "Paradigmas, métodos y técnicas" (Tomo I, pp. 202-204). Buenos Aires: Facultad 
de Psicología de la Universidad de Buenos Aires. Recuperado de http://www.fapyd. unr.edu.ar/academica/archivos/seminario_formacion_docente/10_diment_y_carlino_ perspectivas_de_alumnos.pdf

Duras, M. (1994). Escribir. Barcelona: Tusquets.

Fernández, G. y Carlino, P. (2006). Leer y escribir en la escuela media y en la universidad. Diferencias percibidas por ingresantes a la Facultad de Ciencias Humanas de la UNCPBA. En Memorias de las XIII jornadas de investigación en psicología y segundo encuentro de investigadores en psicología del Mercosur "Paradigmas, Métodos y Técnicas (Tomo I, pp. 227-229. Buenos Aires: Facultad de Psicología de la Universidad de Buenos Aires. Recuperado de http:// www.fvet.uba.ar/postgrado/especialidad/blb/Fernandez_y_Carlino_Leer_y_escribir_en_ la_esc_media_y_en_la_universidad_diferencias_Psico_06.pdf

Fontaine, A. (1995). La implantación del significante en el cuerpo. Revista Litoral, 18/19, 9-34.

Foucault, M. (1992). Microfísica del poder. Madrid: La Piqueta.

Habermas, J. (1994). Conocimiento e interés. En J. Habermas (Ed.), Ciencia y técnica como "ideología" (pp. 159-181). Madrid: Editorial Tecnos.

Honneth, A. (2007). Reificación. Un estudio en la teoría del reconocimiento. Buenos Aires: Katz.

Lacan, J. (1997). El seminario. Libro 3. Las psicosis 1955-1956. Buenos Aires: Paidós, 1997.

Merleau-Ponty, M. (1973). The Prose of the World [La prosa del mundo]. Evanston: Northwestern University Press.

Ong, W. J. (1997). Oralidad y escritura. Tecnologías de la palabra. México: Fondo de Cultura Económica.

Pavese, C. (1976). El oficio de vivir. Buenos Aires: Siglo Veinte Editores.

Truffaut, F. (Director). (1959). Les Quatre Cents Coups (Les 400 coups) [Los cuatrocientos golpes]. [Film]. París: Les Films du Carrosse.

Tyler, R. W. (1949). Basic Principles of Curriculum and Instruction. [Principios básicos del currículum y la enseñanza] Chicago: The University of Chicago Press.

(3)

\section{Cómo citar este artículo en APA:}

Cartolari, M. y Zambrano, J. (2013). Notas sobre la escritura escolar como efecto de verdad sobre el sujeto o como gesto en la relación con el saber. Revista Electrónica Educare, 17(1), 129-139. Recuperado de http://www.revistas.una.ac.cr/index.php/EDUCARE/ issue/current

Nota: Para citar este artículo en otros sistemas puede consultar el hipervínculo "Como citar el artículo" en la barra derecha de nuestro sitio web:

http://www.revistas.una.ac.cr/index.php/EDUCARE/index 